\title{
Síndrome de Budd-Chiari por obstrução da via de saída da veia hepática por membrana: tratamento percutâneo por angioplastia com balão
}

\section{Budd-Chiari syndrome due to hepatic venous web outflow obstruction: percutaneous treatment with balloon angioplasty}

Patrick Bastos Metzger ${ }^{1}$ (D), Kamilla Rosales Costa ${ }^{1}$ (D), Simone Lessa e Silva², Valter Ribeiro dos Santos Junior ${ }^{3}$, Vinicius Nunes ${ }^{3}$, Murilo Quadro Berbert Freire ${ }^{3}$, Milton Oliveira de Albuquerque Mello Neto ${ }^{3}$

\begin{abstract}
Resumo
A síndrome de Budd-Chiari é uma doença venosa hepática rara, mais incidente em adultos jovens, podendo se apresentar na forma aguda, subaguda ou crônica, o que resulta em hipertensão portal. O tratamento tradicional consiste em técnicas de trombólise e de shunts portossistêmicos intra-hepáticos, como pontes para o transplante hepático. Recentemente, técnicas de angioplastia com balão ou stents têm sido relatadas para o tratamento dessa afecção. Neste artigo, é relatado e discutido um caso de síndrome de Budd-Chiari por obstrução membranosa da via de saída da veia supra-hepática com trombose da veia hepática média em uma paciente de 24 anos. O tratamento estabelecido foi a angioplastia transjugular com balão, que obteve resultados satisfatórios e boa evolução clínica.
\end{abstract}

Palavras-chave: síndrome de Budd-Chiari; trombose venosa; angioplastia; procedimentos endovasculares.

\begin{abstract}
The Budd-Chiari syndrome is a rare hepatic venous disease. It is more prevalent in young adults and may present in acute, subacute, or chronic forms, causing portal hypertension. Traditional treatment consists of thrombolysis techniques and transjugular intrahepatic portosystemic shunt, as a bridge to liver transplantation. Recently, use of balloon or stent angioplasty techniques has been reported for treatment of this condition. In this article, we report and discuss a case of BCS by membranous obstruction in the hepatic vein outflow tract, with middle hepatic vein thrombosis, in a 24-year-old patient. The treatment chosen and employed was transjugular balloon angioplasty, which achieved satisfactory results and good clinical evolution.
\end{abstract}

Keywords: Budd-Chiari syndrome; venous thrombosis; angioplasty; endovascular procedures.

Como citar: Metzger PB, Costa KR, Silva SL, et al. Síndrome de Budd-Chiari por obstrução da via de saída da veia hepática por membrana: tratamento percutâneo por angioplastia com balão. J Vasc Bras. 2021;20:e20200133. https:// doi.org/10.1590/1677-5449.200133 


\section{INTRODUÇÃO}

A síndrome de Budd-Chiari (SBC) primária é uma doença vascular hepática extremamente rara, decorrente da obstrução do fluxo venoso de saída hepático, geralmente ao nível da veia hepática $(\mathrm{VH})$ ou da veia cava inferior $(\mathrm{VCI})^{1-3}$. Os dados epidemiológicos globais da SBC são escassos e estimam que a sua incidência seja cerca de um caso por milhão de habitantes por ano, considerando estudos epidemiológicos nos continentes asiático e europeu ${ }^{1}$.

A etiopatogênese da SBC difere entre países orientais e ocidentais. Na maioria dos países ocidentais, a principal etiologia da SBC é trombótica, e, por isso, as principais formas de tratamento são a trombólise e o shunt trans-hepático portossistêmico (TIPS), como pontes para o transplante hepático. Já na China e em diversos outros países orientais, a principal causa é a doença venosa oclusiva por membrana desde qualquer segmento venoso hepático até a junção cavo-atrial, sendo a angioplastia com ou sem stent a primeira escolha para tratamento ${ }^{2,3}$.

Relatamos o tratamento endovascular de uma SBC por membrana oclusiva na via de saída da veia suprahepática, com repercussão clínica e hemodinâmica, pela venografia via acesso jugular e uso de angioplastia com balão.

O protocolo foi aprovado pelo Comitê de Ética de nossa instituição (parecer número 4.749.464).

\section{DESCRIÇÃO DO CASO}

Paciente do sexo feminino, 24 anos, previamente saudável, raça branca, natural e procedente de Feira de Santana (BA), com história de distensão abdominal, prostração e um episódio de hematêmese havia 3 meses. A paciente não apresentava histórico de traumas, tromboses prévias, gestação, dor abdominal, icterícia, hematomas ou acolia fecal, assim como negava qualquer descendência oriental. $\mathrm{O}$ exame clínico confirmou a distensão abdominal, com veias colaterais abdominais, hepatomegalia e ascite. $\mathrm{O}$ exame laboratorial revelou enzimas hepáticas, provas de coagulação e bilirrubina dentro da normalidade e albumina sérica baixa. O exame ultrassonográfico revelou ascite com fluxo sanguíneo portal hepatopedal normal. A tomografia computadorizada (TC) com contraste revelou hepatomegalia com realce heterogêneo, denominado fígado em "noz-moscada", lobo caudado hipertrofiado, e as veias hepáticas direita e esquerda apresentavam retenção de contraste. Não houve boa visualização da VH média, sugerindo trombose (Figura 1A). A paciente foi diagnosticada com SBC por provável obstrução da via de saída da veia supra-hepática, sendo encaminhado à radiologia intervencionista para venografia e tratamento, após endoscopia digestiva alta com escleroterapia endoscópica das varizes esofágicas.

A venografia foi realizada sob anestesia local e analgesia com sedação consciente, utilizando abordagem transjugular, mas houve dificuldade de cateterização da veia supra-hepática. Foi, então, utilizado o ultrassom intravascular (UI) para melhor visualização da desembocadura da VH na VCI. O UI confirmou uma estenose $>80 \%$ na via de saída das VHs, direita e esquerda, e a flebografia demonstrou estase venosa dessas duas veias, assim como estenose crítica na via de saída. A VH média não foi visualizada. Um fio guia hidrofílico 0,035 × $260 \mathrm{~mm}$ (Terumo

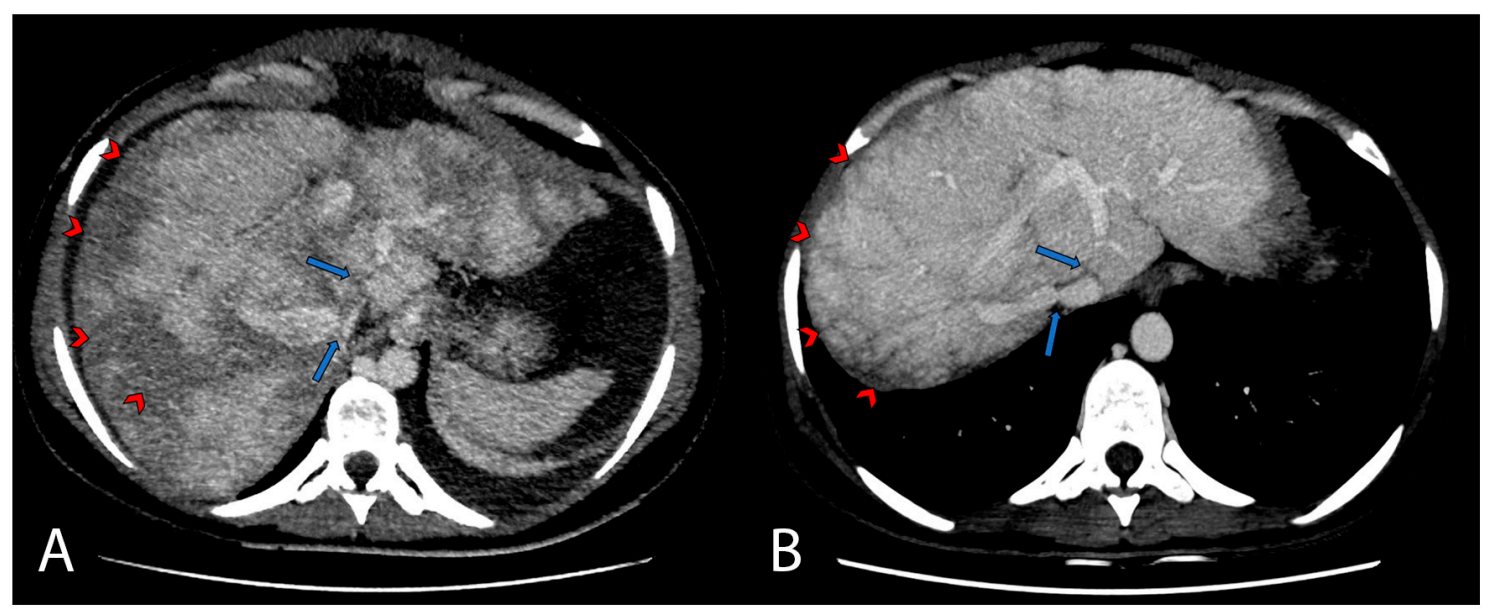

Figura 1. Imagens tomográficas axiais na fase portal do pré-operatório da primeira intervenção em (A) e antes da segunda intervenção (B) endovascular (2 anos e 8 meses após), demonstrando a evolução das estenoses nas vias de saídas nas veias hepáticas (setas longas) e da alteração na proporção dos distúrbio perfusional do parênquima hepático (cabeça de seta). Nota-se também a não opacificação da veia hepática média. 
Medical, Somerset, NJ) foi, então, introduzido através da estenose crítica da veia supra-hepática, via veia jugular direita. A angioplastia da estenose com balões de alta pressão de $8 \mathrm{~mm}$, inicialmente, e $12 \mathrm{~mm}$ posteriormente restabeleceu o fluxo (Figura 2). A paciente evoluiu satisfatoriamente no pós-operatório, com acentuada redução na circunferência abdominal e dos vasos colaterais abdominais, tendo alta hospitalar com 15 dias de pós-operatório, em uso de rivaroxabana, com a intenção de uso por 6 meses.

Durante o acompanhamento pós-operatório com 1,3 e 12 meses, apresentou evolução favorável e sem sinais de síndrome de hipertensão portal (SHP) nos exames clínicos, laboratoriais e de imagem. Foram feitos exames para trombofilia, após a suspensão da anticoagulação, com resultados normais. Após 2 anos de acompanhamento, a paciente retornou em consulta ambulatorial grávida de 14 semanas, sem sinais de descompensação clínica ou laboratorial, sendo introduzida anticoagulação com heparina fracionada pela gastroenterologia e hematologia. A gestação veio a termo com 38 semanas, e, no puerpério, a paciente manteve evolução favorável, sendo mantida a anticoagulação. Após o puerpério, realizou nova TC que demonstrou uma diminuição do diâmetro da via de saída $(7 \mathrm{~mm})$ comparada ao exame prévio
(12 mm), com o aparecimento de ascite detectada pela TC, porém sem evidências clínicas (Figura 1B). Optado, em conjunto com a gastro-hepatologia, por nova angioplastia da veia supra-hepática.

$\mathrm{O}$ procedimento foi realizado pela mesma via e utilizando os procedimentos anestésicos anteriormente descritos, sendo evidenciada estenose na via de saída de cerca de $50 \%$ pela flebografia. Foram realizadas angioplastias consecutivas com balões venosos de alta pressão de 10 e $14 \mathrm{~mm}$. O resultado venográfico foi satisfatório, e a alta hospitalar foi realizada no dia seguinte ao procedimento (Figura 3). A TC de controle com 1 mês de pós-operatório demonstrou uma melhora do diâmetro da via saída (12 $\mathrm{mm})$ e ausência de ascite. Atualmente encontra-se em acompanhamento, com 1 ano de pós-operatório, sendo realizado ultrassom abdominal total (USG) e dúplex das veias suprahepáticas a cada 6 meses, estando assintomática e com exames laboratoriais normais. A paciente deu consentimento para a descrição e publicação do caso.

\section{DISCUSSÃO}

A SBC constitui uma afecção de incidência extremamente baixa. Os dados epidemiológicos são escassos, e, quando encontrados, os estudos são em sua maioria realizados no continente asiático ${ }^{1}$.
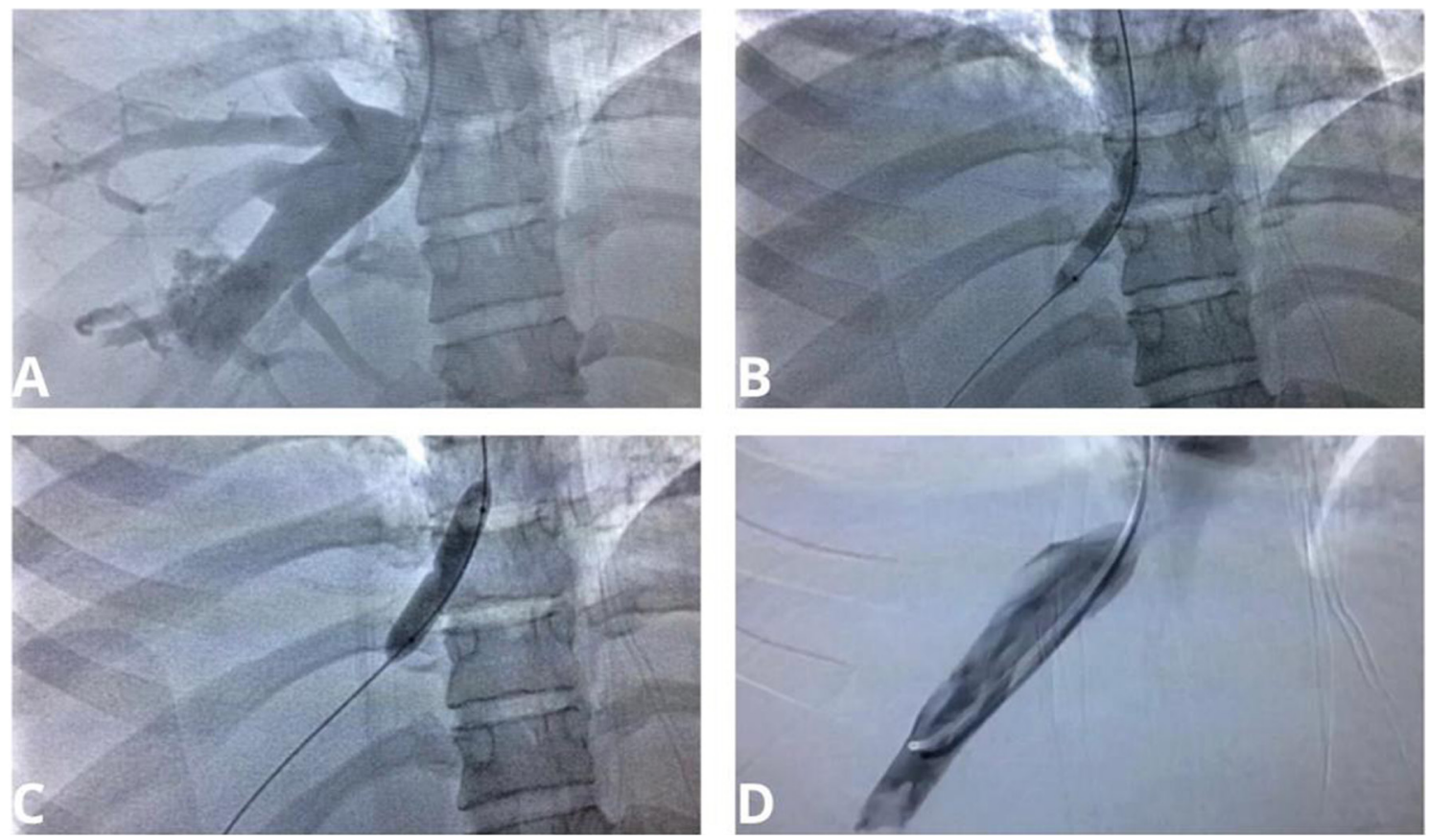

Figura 2. Imagens da flebografia intraoperatória de veia supra-hepática seguida de angioplastia com balão na primeira intervenção. Em (A), flebografia demonstrando estase de veias hepáticas, ocasionada por estenose em via de saída da veia supra- hepática. Em (B), pré-dilatação com uso de balão de $8 \mathrm{~mm}$. Observe a estenose ocasionada no balão. Em (C), dilatação com balão de $12 \mathrm{~mm}$. Observe a diminuição da estenose ocasionada no balão. Em (D), flebografia de controle demonstrando melhora importante do fluxo na via de saída. 

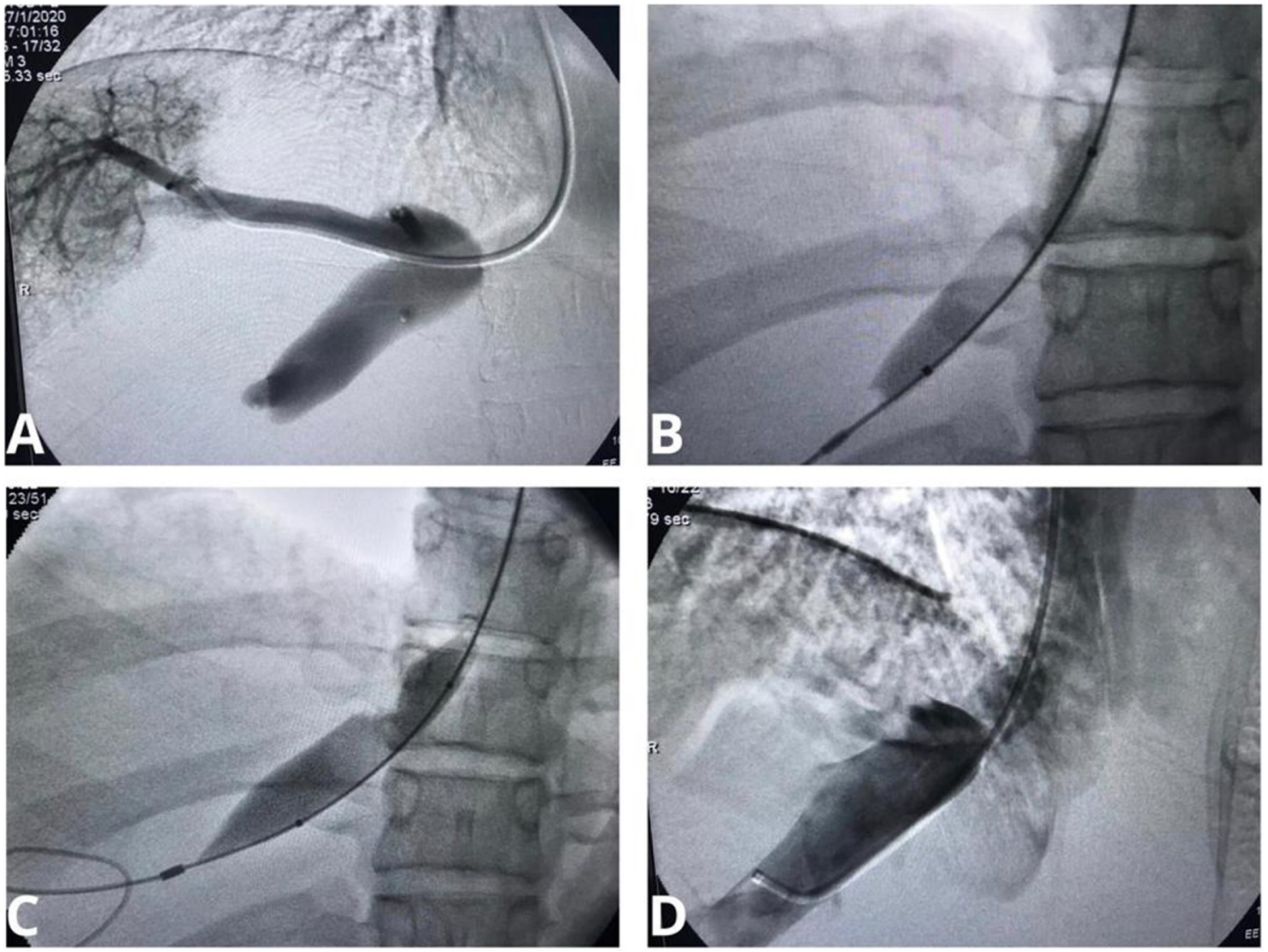

Figura 3. Imagens da flebografia intraoperatória de veia supra-hepática seguida de angioplastia com balão na segunda intervenção. Em (A), flebografia demonstrando estase de veias hepáticas, ocasionada por estenose em via de saída da veia supra- hepática. Em (B), pré-dilatação com uso de balão de $10 \mathrm{~mm}$. Observe a estenose ocasionada no balão. Em (C), dilatação com balão de $14 \mathrm{~mm}$. Em (D), flebografia de controle demonstrando melhora importante do fluxo na via de saída.

Essa síndrome é causada pela oclusão do fluxo venoso hepático a qualquer nível desde as VHs até a junção átrio-caval, podendo ser de origem primária ou secundária. A trombose intraluminal intrínseca constitui a principal causa primária da SBC, mais frequente em adultos jovens e com anormalidade hematológica subjacente, que desencadeia um estado de hipercoagulabilidade. Algumas das afecções que originam o estado de hipercoagulação são doença mieloproliferativa, deficiência de proteína $\mathrm{C}$ ou $\mathrm{S}$, trombocitose essencial, lúpus eritematoso sistêmico, mutação do fator V Leiden, hemoglobinúria paroxística noturna, síndrome antifosfolipídica e uso de contraceptivos orais. Já as causas secundárias podem ser representadas por compressão extraluminal (abscesso ou tumor) ou ainda por invasão intraluminal (tumoral ou parasitária) $)^{2-5}$.

A fisiopatologia da formação da trombose intraluminal não é bem conhecida, mas a principal suspeita é de um processo multifatorial que envolve o constante movimento respiratório do diafragma associado a uma confluência perpendicular da veia supra-hepática à VCI, que causa lesões endoteliais microscópicas na VCI em seu segmento supra-hepático. Além disso, na população ocidental diagnosticada com SBC, é possível observar uma forte associação com um estado de hipercoagulabilidade que promove a microtrombose. Esse processo é lento e gradual, levando a uma organização de tecido fibrótico na parede da veia. Já nos pacientes orientais, é incomum identificar anormalidades hematológicas subjacentes, evidenciando diferenças relevantes da etiologia dessa síndrome entre as distintas populações, sendo a presença de membranas a principal causa para a SBC nessa população ${ }^{5}$.

A SBC pode ser classificada de acordo com o seu tempo de evolução e gravidade, em fulminante, aguda, subaguda ou crônica ${ }^{2}$. Outra classificação utilizada é de acordo com o local de acometimento da obstrução: tipo VCI (acomete apenas a VCI, sem comprometimento da $\mathrm{VH}$ ), tipo $\mathrm{VH}$ (acometimento da $\mathrm{VH}$, incluindo a VH acessória, mas sem comprometimento da 
VCI) e tipo combinado (comprometimento da VCI e pelo menos uma das tributárias da $\mathrm{VH})^{5}$. Essas classificações são importantes no planejamento da abordagem terapêutica. $\mathrm{O}$ caso relatado trata-se de uma obstrução primária, sem disfunção de coagulabilidade subjacente conhecida, classificada como crônica e do tipo $\mathrm{VH}$, como evidenciado pelo quadro clínico e pela TC com contraste.

A formação da membrana obstrutiva no interior da veia é um processo lento que apresenta compensação através do estabelecimento de circulação colateral. Os sintomas só se tornam presentes nos pacientes quando a estenose é significativa, permanecendo assintomático até esse momento ${ }^{5}$. No entanto, a apresentação clínica depende ainda da extensão e rapidez da obstrução do fluxo de saída, podendo manifestar-se em poucos dias ou após anos de evolução ${ }^{4}$. Os sintomas são progressivos até cirrose hepática, quando não tratada em tempo hábil ${ }^{6}$. Quando instaurado o quadro agudo, pode culminar em insuficiência hepática, manifestando-se com náuseas, vômitos, icterícia e alterações laboratoriais ${ }^{2,6}$. Já o quadro crônico pode caracterizar-se pela presença de hepatomegalia, icterícia e SHP, além de complicações mais tardias, como sangramento do trato gastrointestinal e carcinoma hepatocelular ${ }^{2,4,6}$.

$\mathrm{O}$ caso relatado evidencia uma paciente jovem, previamente saudável, com queixas crônicas de distensão abdominal, prostração e um episódio de hematêmese. Ao exame físico, apresentou os achados clínicos de SHP. A SBC deve constituir um dos diagnósticos diferenciais para todo paciente que apresentar esses achados na ausência de doença parenquimatosa hepática primária ou secundária ${ }^{7}$.

As opções de tratamento são anticoagulação, terapia endovascular de descompressão (trombólise, angioplastia ou shunt portossistêmico intra-hepático transjugular) ou transplante de fígado. A evolução das possibilidades de tratamento para a SBC possibilitou um importante aumento da sobrevida desses pacientes $(80-90 \%)^{6}$.

A angioplastia com balão ou stent é a primeira linha para o tratamento da SBC primária por membrana obstrutiva, e sua eficácia é de $95,7 \% \%^{3,6,8-10}$. Pode ser realizada por via jugular, hepática percutânea ou femoral; no entanto, em alguns casos se faz necessária a abordagem combinada ${ }^{3,7-10}$. A via de acesso escolhida para a abordagem da paciente em questão foi a via jugular, que apresenta alta taxa de sucesso, pois o ângulo entre a VH e a VCI é relativamente grande, facilitando a passagem do fio-guia ${ }^{6}$. Após a passagem do fio-guia pelo segmento ocluído, é realizada a dilatação do segmento estenosado com o uso do balão de alta pressão com diâmetros progressivamente maiores ${ }^{3}$. O uso do balonamento progressivo, assim como a insuflação e desinsuflação lenta dos balões, deve ser realizado consoante às medidas tomográficas pré-operatórias e também conforme a sintomatologia de dor da paciente - uma vez havendo essa queixa, pressão maior no balão não deve ser alcançada. O uso do stent é reservado para lesões que não melhoram o gradiente pressórico com a angioplastia com balão ou que apresentam recoil importante, mesmo após balonamentos progressivos. No caso apresentado, após a dilatação do segmento estenosado, foi possível notar a melhora do quadro clínico de forma precoce, com redução da ascite, do edema e dos vasos colaterais abdominais ${ }^{3}$.

Após o tratamento endovascular, faz-se necessário o acompanhamento do paciente com avaliação clínica e realização de exames de imagem com USG com Doppler e $\mathrm{TC}^{2}$, além de manutenção de anticoagulação por pelo menos 6 meses após a angioplastia, podendo esse período ser variável de acordo com as condições do paciente ${ }^{6}$. A paciente apresentou uma nova estenose após 2,5 anos do tratamento endovascular inicial, logo após o período de puerpério. Essa condição fisiológica é responsável pelo estabelecimento de um estado de hipercoagulabilidade a partir da $10^{\mathrm{a}}$ semana de gestação, o que pode agir como determinante na formação de nova membrana obstrutiva da $\mathrm{VH}$ e consequente trombose ${ }^{3,11}$. Portanto, tornam-se necessários a anticoagulação e o acompanhamento rigoroso do caso, para que, em uma eventual descompensação clínica, medidas como nova angioplastia com balão ou com stent, em caso de falha, estejam prontamente disponíveis.

\section{REFERÊNCIAS}

1. Li Y, De Stefano V, Li H, et al. Epidemiology of Budd-Chiari syndrome: a systematic review and meta-analysis. Clin Res Hepatol Gastroenterol. 2019;43(4):468-74. http://dx.doi.org/10.1016/j. clinre.2018.10.014. PMid:30528513.

2. Bozorgmanesh A, Selvam DA, Caridi JG. Budd-Chiari syndrome: hepatic venous web outflow obstruction treated by percutaneous placement of hepatic vein stent. Semin Intervent Radiol. 2007;24(1):100-5. http://dx.doi.org/10.1055/s-2007-971200. PMid:21326746.

3. Mukund A, Gamanagatti S. Imaging and interventions in BuddChiari syndrome. World J Radiol. 2011;3(7):169-77. http://dx.doi. org/10.4329/wjr.v3.i7.169. PMid:21860712.

4. Bayraktar UD, Seren S, Bayraktar Y. Hepatic venous outflow obstruction: three similar syndromes. World J Gastroenterol. 2007;13(13):1912-27. http://dx.doi.org/10.3748/wjg.v13.i13.1912. PMid:17461490.

5. Ding PX, Liu C, Han XW, Ding JY, Tse G, Lee EW. Obstructed membranous transformation of the inferior vena cava in patients with hepatic vein-type Budd-Chiari syndrome: a case series. Clin Res Hepatol Gastroenterol. 2020;44(2):e17-24. http://dx.doi. org/10.1016/j.clinre.2019.10.008. PMid:31786160. 
6. Cheng DL, Zhu N, Xu H, et al. Outcomes of endovascular interventional therapy for primary Budd-Chiari syndrome caused by hepatic venous obstruction. Exp Ther Med. 2018;16(5):4141-9. http://dx.doi.org/10.3892/etm.2018.6708. PMid:30402156.

7. Sparano J, Chang J, Trasi S, Bonanno C. Treatment of the BuddChiari syndrome with percutaneous transluminal angioplasty: case report and review of the literature. Am J Med. 1987;82(4):821-8. http://dx.doi.org/10.1016/0002-9343(87)90022-2. PMid:2952007.

8. Lee BB, Villavicencio L, Kim YW, et al. Primary Budd-Chiari syndrome: outcome of endovascular management for suprahepatic venous obstruction. J Vasc Surg. 2006;43(1):101-8. http://dx.doi. org/10.1016/j.jvs.2005.09.003. PMid:16414396.

9. Qi X, Han G, Guo X, et al. Review article: the aetiology of primary Budd-Chiari syndrome: differences between the West and China. Aliment Pharmacol Ther. 2016;44(11-12):1152-67. http://dx.doi. org/10.1111/apt.13815. PMid:27734511.

10. Valla DC. Budd-Chiari syndrome/hepatic venous outflow tract obstruction. Hepatol Int. 2018;12(Supl 1):168-80. http://dx.doi. org/10.1007/s12072-017-9810-5. PMid:28685257.

11. Souza Al, Batista M Fo, Ferreira LOC. Alterações hematológicas e gravidez. Rev Bras Hematol Hemoter. 2002;24(1):29-36. http:// dx.doi.org/10.1590/S1516-84842002000100006.
Correspondência Patrick Bastos Metzger Escola Bahiana de Medicina e Saúde Pública - EBMSP Rua Professor Diógenes Rebouças, 128 CEP 41830-570 - Salvador (BA), Brasil Tel.: (71) 99977-6356 E-mail: patrickvascular@gmail.com

Informações sobre os autores PBM - Doutor em Medicina, Universidade de São Paulo; Cirurgião Vascular e Endovascular, Sociedade Brasileira de Angiologia e Cirurgia Vascular (SBACV); Radiologista Intervencionista, Colégio Brasileiro de Radiologia (CBR).

KRC - Acadêmica do $9^{\circ}$ semestre de Medicina, Escola Bahiana de Medicina e Saúde Pública (EBMSP).

SLS - Preceptora, residência de Geriatria, Hospital Santo Antônio. VRS - Radiologista, Clínica Delfin, Hospital da Bahia (HBA).

VN - Hepatologista, Hospital da Bahia (HBA), Hospital Aliança e Clínica AMO.

MQBF e MOAMN - Cirurgiões Vascular e Endovascular, Sociedade Brasileira de Angiologia e Cirurgia Vascular (SBACV).

Contribuições dos autores Concepção e desenho do estudo: PBM Análise e interpretação dos dados: KRC, PBM Coleta de dados: PBM, MQBF, MOAMN Redação do artigo: KRC, PBM, SLS, VRS)

Revisão crítica do texto: PBM Aprovação final do artigo*: KRC, PBM, SLS, VRSJ, VN, MQBF, MOAMN

Análise estatística: KRC, PBM Responsabilidade geral pelo estudo: KRC, PBM, SLS, VRSJ, VN

*Todos os autores leram e aprovaram a versão final submetida ao J Vasc Bras. 